\title{
Shear wave splitting and crustal anisotropy at the Mid-Atlantic Ridge, $35^{\circ} \mathrm{N}$
}

\author{
Andrew H. Barclay \\ School of Oceanography, University of Washington, Seattle, Washington, USA
}

Douglas R. Toomey

Department of Geological Sciences, University of Oregon, Eugene, Oregon, USA

Received 10 August 2001; revised 27 February 2003; accepted 23 April 2003; published 14 August 2003.

[1] Shear wave splitting observed in microearthquake data at the axis of the Mid-Atlantic Ridge near $35^{\circ} \mathrm{N}$ has a fast polarization direction that is parallel to the trend of the axial valley. The time delays between fast and slow $S$ wave arrivals range from 35 to $180 \mathrm{~ms}$, with an average of $90 \mathrm{~ms}$, and show no relationship with ray path length, source-toreceiver azimuth, or receiver location. The anisotropy is attributed to a shallow distribution of vertical, fluid-filled cracks, aligned parallel to the trend of the axial valley. Joint modeling of the shear wave anisotropy and coincident $P$ wave anisotropy results, using recent theoretical models for the elasticity of a porous medium with aligned cracks, suggests that the crack distribution that causes the observed $P$ wave anisotropy can account for at most $10 \mathrm{~ms}$ of the shear wave delay. Most of the shear wave delay thus likely accrues within the shallowmost $500 \mathrm{~m}$ (seismic layer 2A), and the percent $S$ wave anisotropy within this highly fissured layer is $8-30 \%$. Isolated, fluid-filled cracks at $500 \mathrm{~m}$ to $3 \mathrm{~km}$ depth that are too thin or too shallow to be detected by the $P$ wave experiment may also contribute to the shear wave delays. The joint analysis of $P$ and $S$ wave anisotropy is an important approach for constraining the crack distributions in the upper oceanic crust and is especially suited for seismically active hydrothermal systems at slow and intermediate spreading mid-ocean ridges. INDEX TERMS: 3035 Marine Geology and Geophysics: Midocean ridge processes; 7203 Seismology: Body wave propagation; 7220 Seismology: Oceanic crust; KEYWORDS: shear wave splitting, Mid-Atlantic Ridge, crustal anisotropy

Citation: Barclay, A. H., and D. R. Toomey, Shear wave splitting and crustal anisotropy at the Mid-Atlantic Ridge, $35^{\circ}$ N, J. Geophys. Res., 108(B8), 2378, doi:10.1029/2001JB000918, 2003.

\section{Introduction}

[2] The strong axis-parallel alignment of faults and fissures and volcanic ridges observed at the seafloor of midocean ridges indicates that much of the oceanic crust is formed and altered in a predominantly extensional stress field. This stress field will also cause a preferred average alignment of joints, pores, and microcracks that may give rise to anisotropic permeability and so influence the circulation of hydrothermal fluids in the crust and the distribution of hydrothermal vents [Kleinrock and Humphris, 1996b]. Knowing the depth extent of these cracks and the degree of connectedness and alignment is therefore crucial for understanding mid-ocean ridge processes.

[3] The anisotropic propagation of seismic $P$ and $S$ waves can be used to address the nature of aligned crack distributions at depth. For vertical cracks, seismic $P$ waves have an azimuthal velocity variation, with the fastest direction in the plane of the cracks, while $S$ waves generally split into a fast and slow component, with particle motions respectively in

Copyright 2003 by the American Geophysical Union. 0148-0227/03/2001JB000918\$09.00 and normal to the plane of the cracks. $P$ wave velocity variations have primarily been used to study crustal anisotropy in the oceans [Shearer and Orcutt, 1985; Stephen, 1985; Sohn et al., 1997; Barclay et al., 1998; Detrick et al., 1998; Dunn and Toomey, 2001]. A common result of these studies is that anisotropy is restricted to the upper $3 \mathrm{~km}$, with cracks aligned perpendicular to the spreading direction. Although studies of shear wave splitting are common on land, few observations have been made in the oceans [Hung and Forsyth, 1999; Almendros et al., 2000].

[4] Separate $P$ and $S$ wave studies of anisotropy give limited information on the crustal crack distribution. $P$ wave refraction or tomography studies give good depth resolution of the anisotropy except for the shallowmost crust and are not strongly sensitive to thin, isolated, fluid-filled cracks. $S$ waves, by contrast, are influenced by all cracks but give little information on the depth extent of the anisotropy. Coincident $P$ and $S$ wave studies can be used to overcome these restrictions but such studies are rare because crustal earthquake experiments in the oceanic crust and high-quality $P$ wave refraction surveys on land are difficult. In this paper we analyze the shear wave splitting from microearthquake arrivals in the inner valley of the Mid-Atlantic Ridge 


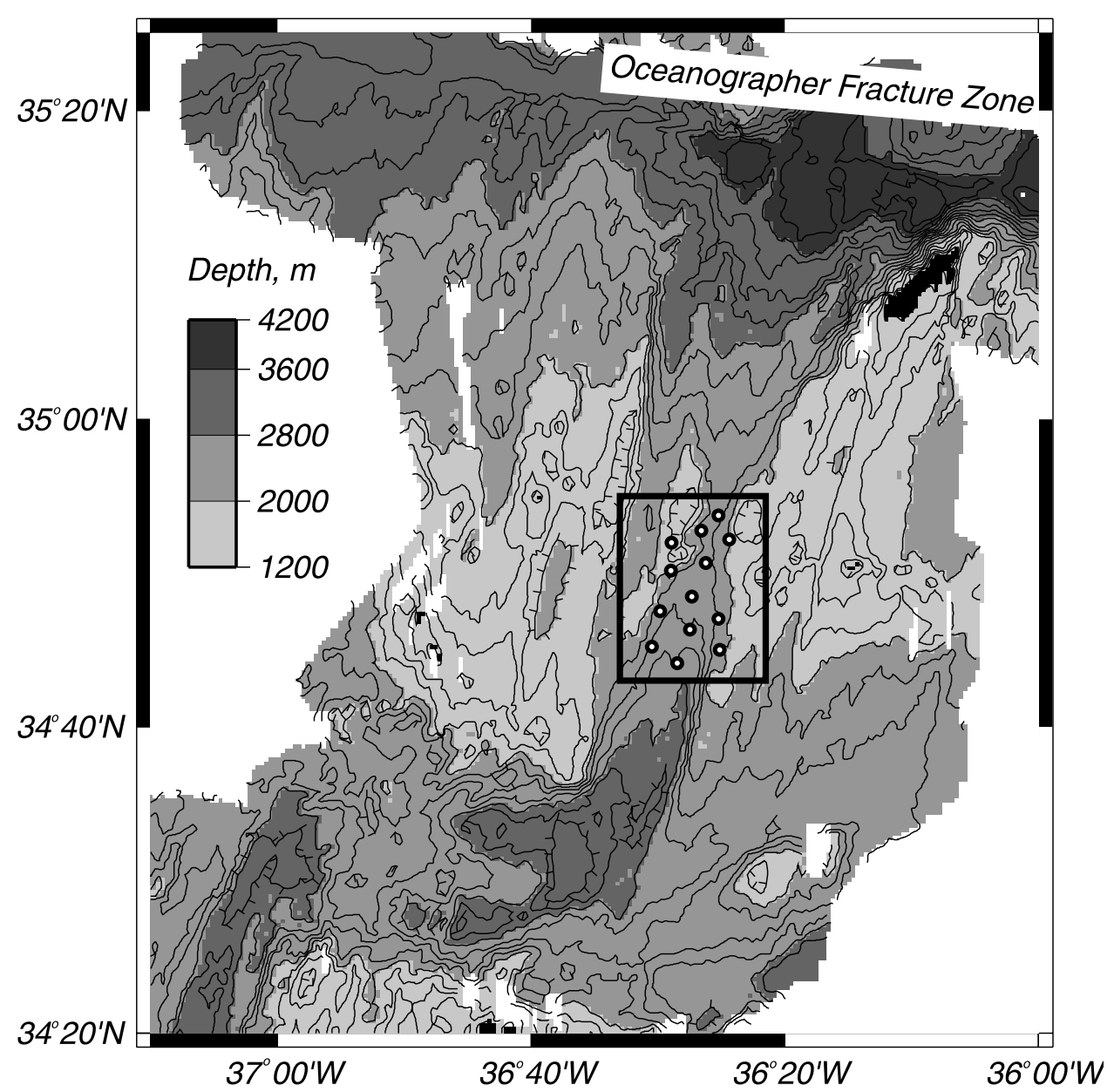

Figure 1. Bathymetry of the MAR segment near $35^{\circ} \mathrm{N}$. The box delimits the area of the microearthquake experiment and of Figure 2. Circles are OBS locations. Contour interval is $200 \mathrm{~m}$; shading changes every $800 \mathrm{~m}$.

(MAR), and measure fast polarization directions and time delays. We jointly interpret our results with observations of the $P$ wave anisotropy at the same site [Barclay et al., 1998] in order to infer the primary causes of anisotropy in the upper oceanic crust.

\section{Method and Results}

[5] The seismic experiment comprised fourteen $1-\mathrm{Hz}$ ocean bottom seismometers (OBS) that were deployed within and around the inner valley of the MAR at $35^{\circ} \mathrm{N}$ (Figure 1). The instruments were spaced $4 \mathrm{~km}$ apart and covered an area approximately 18 by $10 \mathrm{~km}^{2}$. The six OBSs used in this study recorded 1831 air gun shots during the first day of the experiment and then microearthquakes for 42 days. These OBSs also recorded high-quality shear wave arrivals on horizontal components that were level on the seafloor (Figure 2). The orientation of each component pair was determined from the horizontal particle motion of the direct water wave arrivals from the air guns. Further details of the active source tomography and microearthquake experiments are described elsewhere [Barclay et al., 1998, 2001].

[6] We analyzed seismograms from 100 segment center microearthquakes for evidence of shear wave anisotropy.
These earthquakes were used because many of them showed clear, impulsive $S$ wave arrivals and their hypocenters were well constrained. The hypocenters lie at 3-4 km depth beneath the seafloor, are inside or within one station spacing of the OBS array, and form an along-axis band, $12 \mathrm{~km}$ in length, within the inner valley floor, as shown in Figure 2. Most of the focal mechanisms are consistent with faulting on the east dipping scarp that defines the western wall of the inner valley [Barclay et al., 2001]. Examples of split shear wave arrivals are shown in Figure 3 . In each case the horizontal particle motion is initially linear and polarized at $15-30^{\circ}$ east of north, changing to elliptical motion after $50-200 \mathrm{~ms}$. This behavior is characteristic of a linearly polarized fast arrival followed by the combination of its coda and the slow $S$ wave arrival.

[7] Our analysis was restricted to $S$ waves that arrived within the shear wave window (SWW). The SWW is the range of incidence angles for which the particle motion of $S$ waves is unmodified by free-surface interactions and lies between $0^{\circ}$ and $\sin ^{-1}\left(V_{S} / V_{P}\right)$, where $V_{P}$ and $V_{S}$ are the $P$ and $S$ wave velocities at the seafloor [Nuttli and Whitmore, 1962; Evans, 1984]. The maximum limit of the SWW at our study site is $20^{\circ}$. For the average $V_{P} / V_{S}$ value in seismic layer 2A (the shallowmost $400 \mathrm{~m}$ ) of 2.9 [Barclay et al., 2001]. In order to ensure that our arrivals 


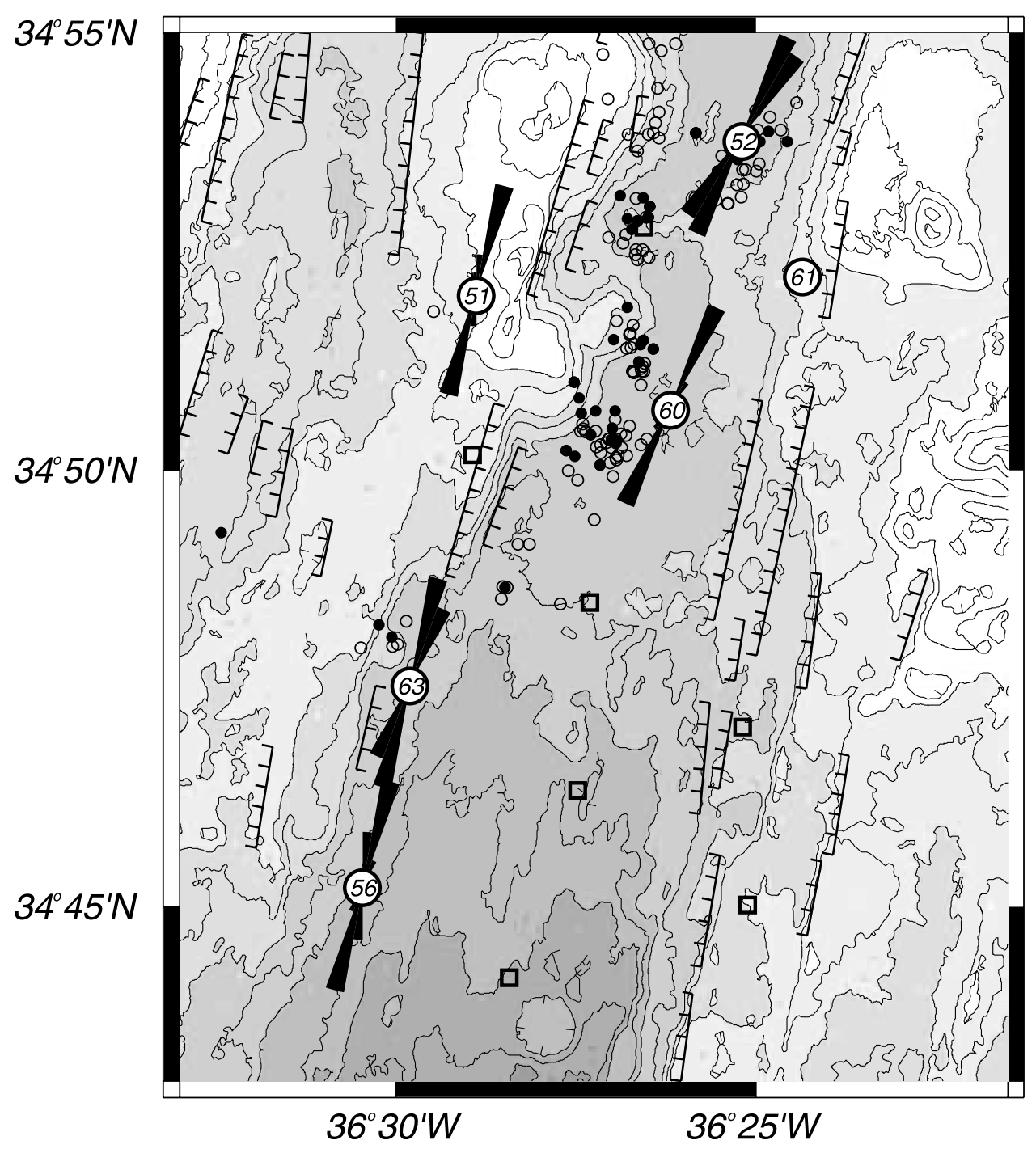

Figure 2. Bathymetry of the along-axis high with rose histograms of fast polarization directions. Squares and circles denote OBS locations and microearthquake epicenters respectively; split shear wave arrivals from 65 earthquakes (solid circles) were observed at six OBSs (numbered squares). Each histogram is normalized to the size of its most populated bin; the number of measurements at each OBS is given in Table 1. Fault scarps are taken from Hekinian et al. [2000]. Contour interval is $50 \mathrm{~m}$.

were within the SWW, we estimated the incidence angle of each arrival by ray tracing through a one-dimensional $S$ wave velocity structure [Barclay et al., 2001]. Layer 2A $S$ wave velocities in this structure were poorly resolved, so we increased the precision of the near-seafloor $S$ wave velocity by dividing the average layer $2 \mathrm{~A} P$ wave velocity of $3.5 \mathrm{~km} / \mathrm{s}$ [Hussenoeder, 1998] by the $V_{P} / V_{S}$ ratio of 2.9. Incidence angles of all of the earthquakes for which shear wave splitting measurements were made are shown in Figure 4. For five OBSs, the incidence angles are within the SWW and for OBS 52 and OBS 60 many arrivals are well within the window. We excluded OBS 61 from our analysis because it lay on a steep slope that dipped toward the hypocenters (Figure 2), causing all $S$ wave arrivals to fall outside the SWW.

[8] The fast polarization directions were measured using polarization analysis [Jurkevics, 1988; Zhang and Schwartz, 1994; Almendros et al., 2000]. By this method, eigenvalue decomposition of the particle motion covariance matrix is used to fit ellipsoids to successive time windows of the particle motion. The polarization direction and incidence angle are measured from $\mathbf{x}_{1}$ and the rectilinearity of the particle motion is defined as $\left|\mathbf{x}_{\mathbf{1}}\right| /\left(\left|\mathbf{x}_{\mathbf{2}}\right|+\left|\mathbf{x}_{\mathbf{3}}\right|\right)$, where $\mathbf{x}_{\mathbf{1}}, \mathbf{x}_{\mathbf{2}}$ and $\mathbf{x}_{\mathbf{3}}$ are the axes of each ellipsoid and $\left|\mathbf{x}_{\mathbf{1}}\right| \geq\left|\mathbf{x}_{\mathbf{2}}\right| \geq\left|\mathbf{x}_{\mathbf{3}}\right|$. An example of the polarization analysis is shown in Figure 5. For each arrival, the polarization was averaged over the interval of high rectilinearity that immediately followed the $S$ wave onset. Having identified arrivals that showed clear evidence of $S$ wave splitting, we rotated the seismograms into fast and slow orientations and measured the time delay between the fast and slow arrivals using cross-correlation and manual inspection. The total number of time delays we measured on the five OBSs (42) was considerably smaller than the number of fast polarization directions (130) because of the difficulty in identifying the slow $S$ wave arrival.

[9] Rose histograms of fast polarization directions for five OBSs are shown in Figure 2, and the results are given 


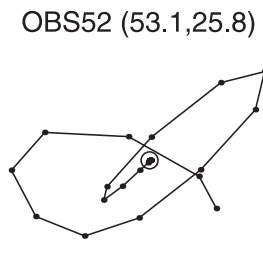

OBS56 $(52.7,26.8)$

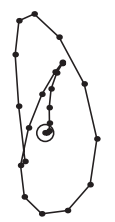

OBS60 $(50.1,26.9)$

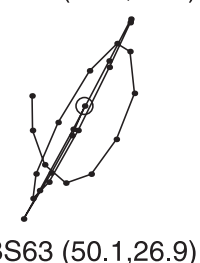

OBS63 $(50.1,26.9)$



OBS52 $(52.4,26.5)$



OBS56 $(49.9,27.5)$

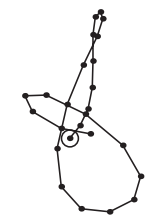

OBS60 $(50.0,27.6)$

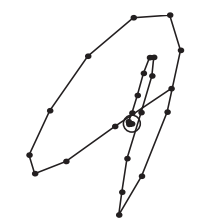

OBS63 $(50.0,27.6)$

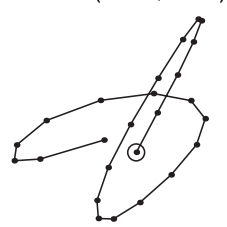

OBS52 $(53.1,25.5)$

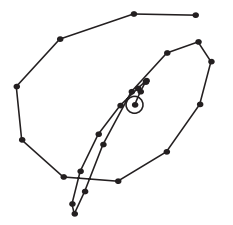

OBS56 $(52.5,26.6)$



OBS60 $(51.2,26.5)$
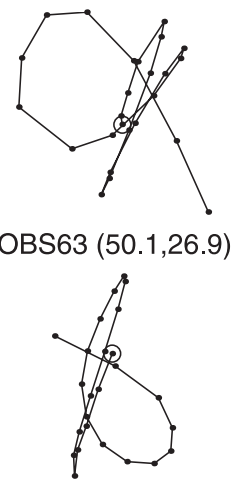

OBS52 $(52.5,26.7)$

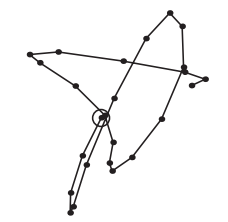

OBS56 (50.3,27.1)

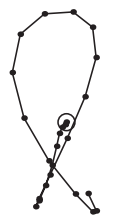

OBS60 (50.3,27.1)

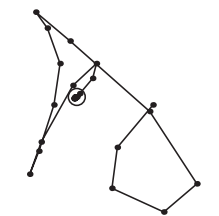

OBS63 (50.2,27.2)

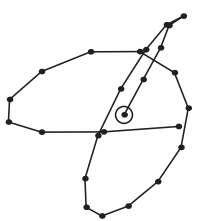

Figure 3. Examples of shear wave splitting in horizontal particle motions. The shear wave arrival is shown for earthquakes at four different OBSs $(52,56,60$, and 63). Numbers in parentheses are the earthquake epicenter in minutes of latitude and longitude, respectively. Each trace is $203 \mathrm{~ms}$ long, with sample points (solid circles) every $7.8125 \mathrm{~s}$. The axes are in the N-S-E-W coordinate system and are normalized to the largest amplitude; the open circle is the origin. The data are unfiltered, and the instrument response is nearly flat from 1 to $30 \mathrm{~Hz}$.

in Table 1. All of the mean directions are within $1 \sigma$ of the trend of the inner valley $\left(015^{\circ}\right)$ except for OBS 52, for which the average fast direction is aligned a further 14 degrees to the east. The time delays range from 35 to $180 \mathrm{~ms}$ (Figure 6), with an average delay of $90 \mathrm{~ms}$. This range is comparable to time delays from other microearthquake studies: 100-300 ms in Iceland [Menke et al., 1994]; 100 to $230 \mathrm{~ms}$ in Hawaii [Munson et al., 1995]; 10-125 ms at the San Andreas Fault [Zhang and Schwartz, 1994]; 40$200 \mathrm{~ms}$ at the Juan de Fuca Ridge [Almendros et al., 2000] and possible split times of $<240 \mathrm{~ms}$ at $26^{\circ} \mathrm{N}$ on the MAR [Kong et al., 1992]. We observed no correlations between the time delay and receiver location, source-receiver azimuth, or source-receiver range (Figure 6).

\section{Interpretation}

[10] The $S$ wave splitting we observe is most likely due to vertical cracks that are preferentially aligned parallel to the trend of the axial valley. Vertical cracks give rise to hexagonal anisotropy with a horizontal symmetry axis that is normal to the crack plane. For this form of anisotropy, fast split $S$ waves are polarized parallel to the crack planes and the slow waves are polarized parallel to the symmetry axis and perpendicular to the fast polarization direction [Crampin, 1993]. This interpretation is supported by the observation of
$P$ wave azimuthal anisotropy at the same site [Barclay et al., 1998] which shows a $\cos (2 \theta) P$ wave velocity variation in the horizontal plane with the fast direction parallel to the trend of the axial valley. Cracks are preferentially aligned perpendicular to the minimum compressive stress direction; the inner valley floor at the MAR is heavily fractured with faults and fissures that strike in the along-axis direction [Ballard and van Andel, 1977; Kleinrock and Humphris, 1996a; Gracia et al., 1999]. On the subaerial Mid-Atlantic Ridge in Iceland, the fast polarization directions of split shear waves are similar to the strikes of surface faults and fissures [Menke et al., 1994].

[11] We discount the possibility that the polarized arrivals are caused by the earthquake source polarizations and that anisotropy is absent, for three reasons. Firstly, the large number and wide global distribution of shear wave splitting observations indicates that crustal anisotropy is ubiquitous [Crampin, 1994]. In addition, the presence of anisotropy beneath the OBS network has already been established by observations of $P$ wave anisotropy [Barclay et al., 1998]. Finally, we confirmed the presence of anisotropy by testing whether the observed fast polarization directions could be explained by the source $S$ wave polarization and propagation through an isotropic medium. For this test we calculated radiation patterns for double couple focal mechanisms [Barclay et al., 2001] and assumed that the source polari- 

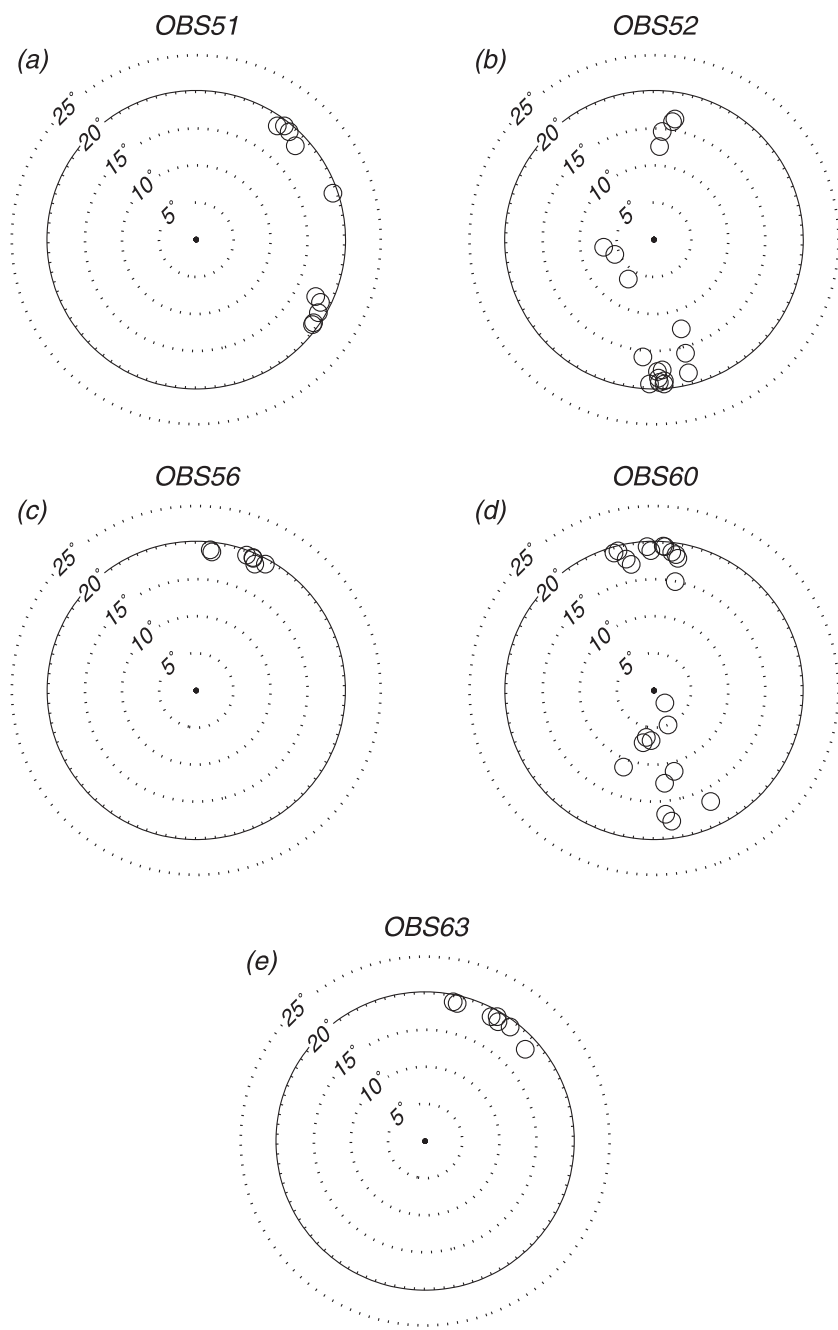

Figure 4. Equal-area projections of the azimuth and incidence angle of $S$ wave arrivals for which fast polarization directions were measured. The solid line is the maximum limit of the shear wave window.

zation was preserved to the surface. Predicted and observed polarizations for two earthquakes are compared in Figures $7 \mathrm{a}$ and $7 \mathrm{~b}$; this comparison was extended to all $S$ wave arrivals for which focal mechanisms and fast polarization measurements were available (Figure 7c). The predicted and observed $S$ wave polarizations in Figure 7 differ significantly and we therefore conclude that anisotropy is required.

[12] The $S$ wave anisotropy is likely restricted to the uppermost $1-2 \mathrm{~km}$ of the oceanic crust. Split $S$ wave delays measured at the seafloor represent the integrated delay due to the anisotropy between the source and receiver, and as our earthquake focal depths were all 3-4 km, we cannot resolve any depth-dependent anisotropy structure. However, the large variation we see in delays for similar path lengths (Figure 6), suggests that the anisotropy is both heterogeneous and shallow, although it is likely that some of the variation is due to difficulties in measuring the delay time between the fast and slow $S$ wave arrivals. This inference has also been made in other crustal $S$ wave splitting studies [e.g., Peacock et al., 1988; Savage et al., 1989; Menke et al., 1994; Zhang and Schwartz, 1994; Munson et al., 1995;
Evans et al., 1996; Almendros et al., 2000]. Additional support for a shallow concentration of anisotropy comes from the depth-dependent $P$ wave anisotropy structure at $35^{\circ} \mathrm{N}$ [Barclay et al., 1998] which decreases from $>4 \%$ at $500 \mathrm{~m}$ depth to zero $P$ wave anisotropy below $1.5 \mathrm{~km}$ depth. We define percentage $P$ wave anisotropy as $100 *\left(V_{\max }-\right.$ $\left.V_{\min }\right) / V_{\text {average}}$, where $V_{\max }, V_{\min }$ and $V_{\text {average }}$ are the maximum, minimum, and direction-averaged wave speeds, and $S$ wave anisotropy as $100 *\left(V_{\max }-V_{\min }\right) / V_{\max }$, following Crampin [1989]. The absence of a variation in time delay with path length for OBS 56 (Figure 6) is also consistent with $S$ wave anisotropy being restricted to the upper $1-2 \mathrm{~km}$. For OBS 56 , the observed $S$ waves propagated approximately in the along-axis direction with path lengths ranging from 11 to $16 \mathrm{~km}$, mostly at depths $>3 \mathrm{~km}$, and all with split shear wave time delays of $\sim 70 \mathrm{~ms}$. Owing to its horizontal, ridgeperpendicular symmetry axis, the anisotropy for along-axis and vertical propagation in a constant anisotropy medium is the same, and so the arrivals at OBS 56 would show different delays, were anisotropy present below $3 \mathrm{~km}$ depth. It is therefore likely that the only anisotropy the $S$ waves arriving at OBS 56 sampled was that in the shallow crust, immediately below the instrument.

[13] The main sources of the anisotropy are likely to be in the extrusive layer and sheeted dikes. Seismic layer 2A, the high-porosity extrusive layer of pillow basalts and sheet flows, is $400-500 \mathrm{~m}$ thick within the inner valley at $35^{\circ} \mathrm{N}$ [Hussenoeder, 1998]. At fast spreading ridges layer 2A is underlain by a $\sim 1$-km-thick section of sheeted dikes that is rooted in gabbro. It is likely that a similar structure exists at the MAR, although the depth and nature of the dike/gabbro transition may be deeper and more gradual [Barclay et al., 1998]. By this interpretation, the $P$ wave anisotropy detected by [Barclay et al., 2001] corresponds to the sheeted dikes, and any anisotropy at shallower depths must reside in the extrusive layer.

[14] Pillow basalts and sheet flows have no preferred vertical orientation, and anisotropy in the extrusive layer is probably due to aligned fissures. Open at the surface, fissures are a common feature of young seafloor [Ballard and van Andel, 1977] and can reach $400 \mathrm{~m}$ depth, or approximately to the base of the extrusive layer. Models for fissure formation include plate extension, contraction of cooling crust and near-surface extension above buried dike tips [Wright, 1998]. Two OBSs (OBS52 and OBS60) were located at the segment center (Figure 2) where the seafloor is covered by fresh, smooth lavas and few fissures or faults [Gracia et al., 1999]. It is likely, however, that these recent flows cover older, fissured seafloor.

[15] Joints within chilled margins are most likely the primary source of anisotropy within the sheeted dikes. Dike-parallel cracks, filled with high-temperature precipitation minerals, are commonly observed in ophiolites and are interpreted as thermal contraction joints that were open at or near the ridge axis. A study of the crack distributions in the Troodos ophiolite indicated that these joints had an average spacing of $1 \mathrm{~m}$, width of $6.3 \mathrm{~mm}$ and aspect ratios of 0.0016 [van Everdingen, 1995]. The mean joint width decreased with increasing depth. Several other studies have measured $\mathrm{P}$ wave azimuthal anisotropy in the sheeted dike section [Shearer and Orcutt, 1985; Stephen, 1985; Sohn et al., 1997; Dunn and Toomey, 2001]. 
(a)

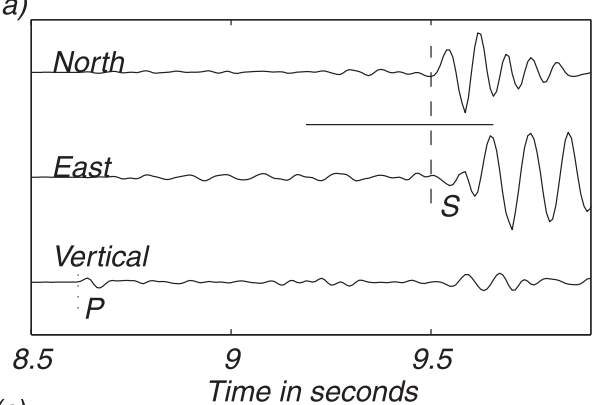

(c)



(b)



(d)

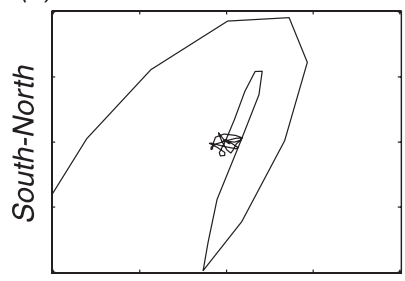

East-West

Figure 5. Example of polarization analysis method. (a) Three-component seismograms before rotation. Seismograms are normalized to the maximum $S$ wave amplitude. The horizontal line shows the duration of the particle motion plot in Figure 5d. The $P$ and $S$ wave onsets are shown by two vertical lines. (b) As for Figure 5a, but after rotation of the horizontal components into fast and slow directions. (c) Polarization (POL), rectilinearity (RECT) and inclination angle (INC) for the arrival in Figures 5a and 5b. The durations of the $P$ and $S$ arrivals are shown by the heavy bars. A running window length of 5 samples was used. See text for details. (d) Horizontal particle motion for the $S$ wave arrival.

[16] Faults and stress-aligned cracks may also contribute to the anisotropy. Three OBSs $(51,56$ and 63$)$ at which we observed shear wave splitting were situated close to the western wall of the inner valley that was the scarp of a large seismically active fault [Barclay et al., 2001]. The other two OBSs were not close to any known faults, and because the time delays measured at the near-fault OBSs were not significantly greater than at the valley floor OBSs, we infer that the presence of the fault does not exert a major control on the anisotropy. Rock samples will become anisotropic when subject to uniaxial stress as microcracks preferentially align perpendicular to the minimum compressive stress direction [Nur and Simmons, 1969]. This effect, called extensive dilatency anisotropy (EDA) has been used to

Table 1. Results of Polarization Analysis ${ }^{\mathrm{a}}$

\begin{tabular}{cccc}
\hline OBS & $\begin{array}{c}\text { Fast Polarization } \\
\text { Direction }\end{array}$ & $\sigma$ & $\begin{array}{c}\text { Number of } \\
\text { Observations }\end{array}$ \\
\hline 51 & 7 & 12 & 20 \\
52 & 29 & 6 & 36 \\
56 & 14 & 8 & 14 \\
60 & 22 & 16 & 46 \\
63 & 20 & 11 & 14 \\
\hline
\end{tabular}

${ }^{\mathrm{a}} \sigma$ is one standard deviation in mean fast polarization direction and includes instrument orientation uncertainties. Directions are degrees clockwise from north explain the widespread occurrence of shear wave splitting observations in the crust [Crampin, 1978]. It is likely that extensional stresses in the highly fissured and porous extrusive layer can be accommodated by the fissures but

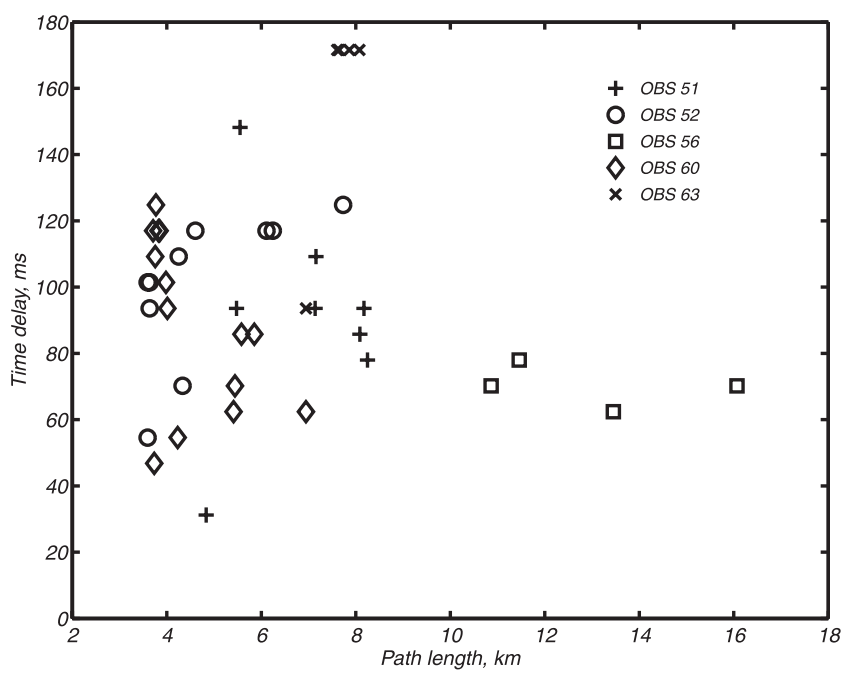

Figure 6. Time delay between fast and slow shear wave arrivals, as a function of source-receiver path length. The symbols represent observations at each of the five OBSs used in this study. 

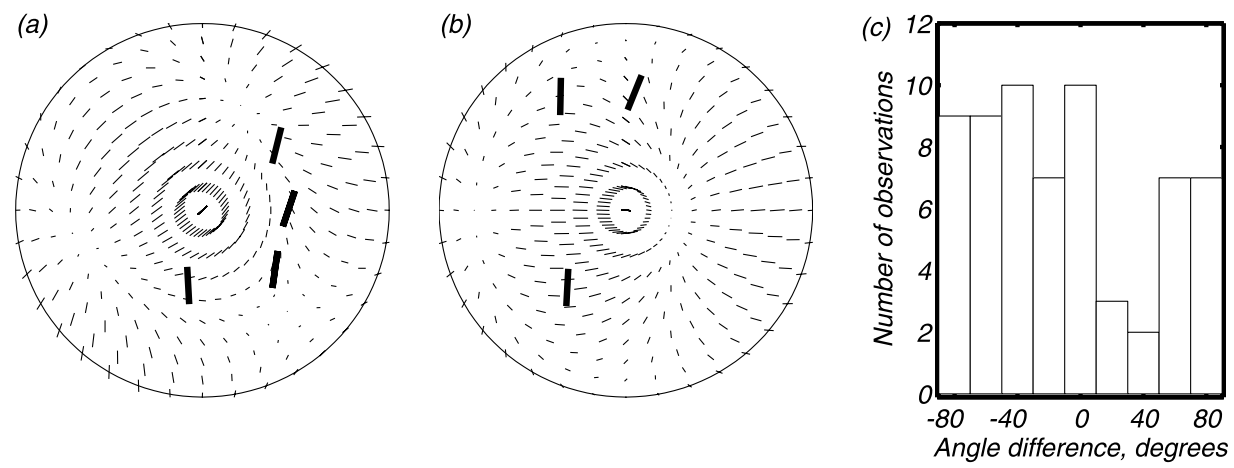

Figure 7. Comparison of predicted $S$ wave source polarizations with observed polarizations. (a) and (b) Equal-area projection of source polarizations for two earthquakes. The light lines show the predicted polarization at the source as a function of takeoff angle and azimuth; the heavy lines show the observed $S$ wave polarization. (c) Distribution of difference between predicted and observed polarizations for all $S$ wave arrivals for which focal mechanisms and fast polarization directions were known.

EDA microcracks may contribute to the anisotropy at deeper depths, as discussed below.

\section{Discussion}

[17] The shear wave splitting and colocated $P$ wave results [Barclay et al., 1998] yield complementary information on the distribution of crack-related anisotropy in the oceanic crust. Each split shear wave delay represents the accrued delay from the earthquake source to the surface and gives no direct information on the depth distribution of anisotropy. The $P$ wave anisotropy, by contrast, is well resolved in depth except for the shallowmost $500 \mathrm{~m}$ where arrivals from turning rays are masked by the direct water wave. In addition, shear waves are nearly insensitive to crack aspect ratios whereas for thin, isolated, fluid-filled cracks the $P$ wave velocity variation is believed to have a $\cos (4 \theta)$ component [Crampin, 1993]. Although no studies of oceanic $P$ wave anisotropy have detected a clear $\cos (4 \theta)$ variation [Shearer and Orcutt, 1985; Stephen, 1985; Sohn et al., 1997], small $\cos (4 \theta)$ signals could be difficult to distinguish from velocity heterogeneity, and it is therefore possible that the shear wave anisotropy samples a distribution of thin, isolated, fluid-filled cracks that $P$ wave studies do not resolve. We explored the significance of these differences by inferring the crack distribution from the $P$ wave anisotropy and estimating its contribution to the shear wave delays.

[18] We selected an effective medium theory for the elastic properties of a medium with aligned cracks and equant pores [Hudson et al., 1996; Pointer et al., 2000]. Although other theoretical formulations exist [e.g., O'Connell and Budiansky, 1974; Nishizawa, 1982; Thomsen, 1995], we used this method because it is an extension of the widely used method of smoothing [Hudson, 1981] and is relatively straightforward to implement. We also chose this approach because it allows for the crack-filling fluid to squirt into surrounding nonaligned pores as the medium is deformed. With the inclusion of this effect, thin fluid-filled cracks (having aspect ratio $<0.05$, and including those observed in the Troodos ophiolite [van Everdingen, 1995]) are predicted to contribute a significant $\cos (2 \theta)$ component to the azimuthal $P$ wave velocity variation that is consistent with the seismic observations. Without considering this effect, thin, isolated, fluid-filled cracks are excluded from the interpretation of the seismic data because only thick, isolated, fluidfilled cracks are predicted to yield a $\cos (2 \theta)$ variation [Hudson, 1981]. Evidence for the presence of a background porosity (equant pores) comes from the low $P$ wave velocities in the uppermost $2 \mathrm{~km}$ of the oceanic crust which cannot be explained by aligned cracks alone [Barclay et al., 1998]. Equant porosity in the extrusive layer is provided by the cracks and voids between pillow lavas and flows; dikeperpendicular cooling joints are a possible source of additional porosity within the sheeted dikes [van Everdingen, 1995].

[19] We used model EQUANT ALIGN [Pointer et al., $2000]$ to obtain the elastic tensor for a particular crack distribution and we used this to calculate the percent $P$ and $S$ wave anisotropies from the eigenvalues of the Christoffel matrix [Babuska and Cara, 1991]. By this model, a single distribution of aligned cracks is parameterized using crack density $\varepsilon$ (defined as $N a^{3} / V$, where $N$ is the number of cracks with maximum radius $a$ in a volume $V$ ) and crack aspect ratio $\gamma=c / a$, where $c$ is the half width of each crack. A model that contains multiple crack populations, specified by a range of aspect ratios and crack densities, is likely to be more representative of the ocean crust, but such a model will not be constrained by our data, thus we assumed a single crack distribution. We also assumed that the anisotropy was a linear function of $\varepsilon$ for $\varepsilon<0.1$ and calculated the anisotropy factor (percent anisotropy / $\varepsilon$ ) for $P$ and $S$ waves and for $\gamma=10^{-1}, 10^{-2}, 10^{-3}$, and $10^{-4}$. Other parameters that we assumed were matrix density and $P$ and $S$ wave velocities of $3000 \mathrm{~kg} / \mathrm{m}^{3}, 6 \mathrm{~km} / \mathrm{s}$ and $3.3 \mathrm{~km} / \mathrm{s}$, respectively. The effect of the equant porosity was parameterized by Pointer et al. [2000] using a dimensionless number, defined as,

$$
P_{e p}=\frac{2 \omega \eta_{f} c^{2}}{\psi_{m} \kappa_{f} K_{m}},
$$

where $\omega$ is the frequency of the seismic wave, $\kappa_{f}$ and $\eta_{f}$ are the fluid bulk modulus and viscosity, and $K_{m}$ and $\varphi_{m}$ are the matrix permeability and porosity respectively. Owing to the 
(a)

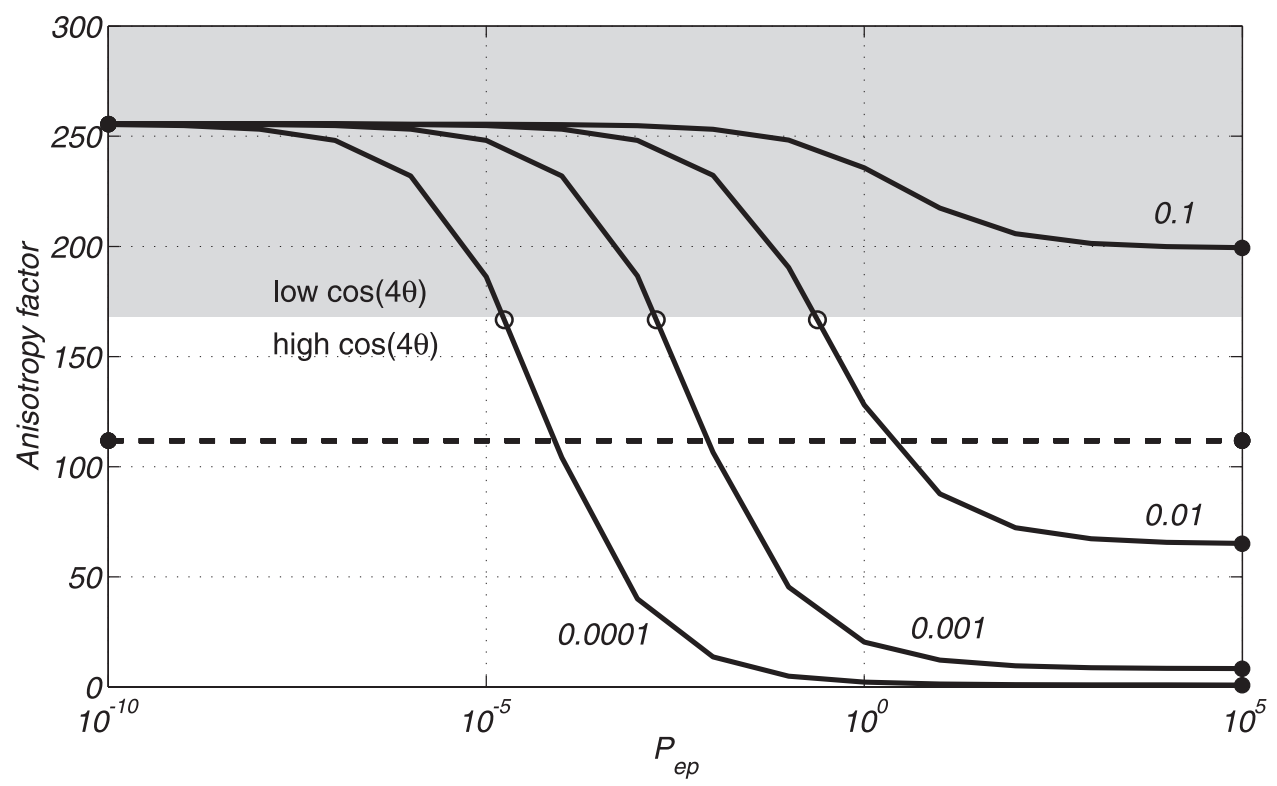

(b)

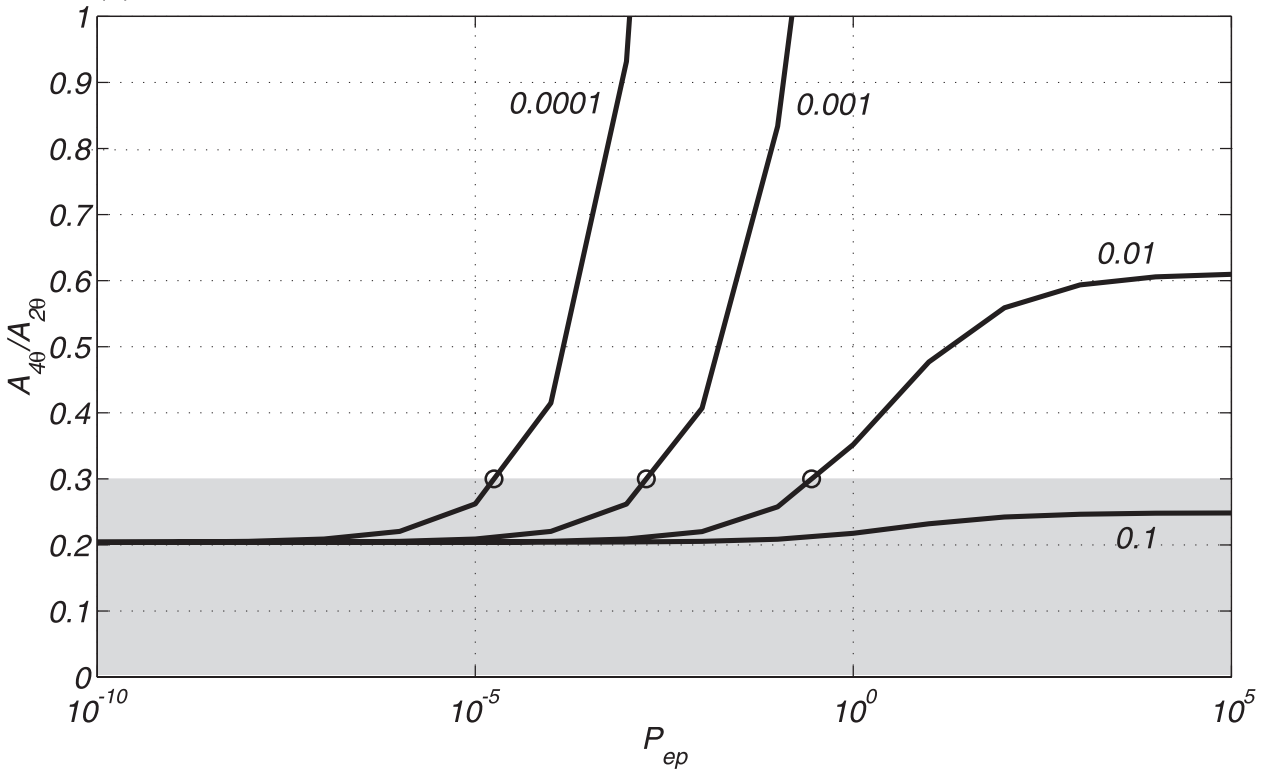

Figure 8. Comparison of $P$ and $S$ wave anisotropies. (a) Predictions of Hudson et al. [1996] for anisotropy factor as a function of parameter $P_{e p}$ [Pointer et al., 2000]. The dashed line is the $S$ wave anisotropy factor for all $\gamma$ and the solid lines are the $P$ wave anisotropy factor for $\gamma=10^{-4}, 10^{-3}, 10^{-2}$, and $10^{-1}$. The solid circles are for the same values of $\gamma$ for the end-member conditions of isolated wet (high $P_{e p}$ ) and dry cracks (low $P_{e p}$ ) [Hudson, 1981]. The $\cos (4 \theta) / \cos (2 \theta)$ amplitude ratio is equal to 0.3 at each of the open circles and $<0.3$ within the shaded region. See text for details. (b) Amplitude ratio of $P$ wave $\cos (4 \theta)$ and $\cos (2 \theta)$ azimuthal velocity variations for the same $\gamma$ and $P_{e p}$ in Figure $8 \mathrm{a}$. For each $\gamma$ an amplitude ratio of 0.3 was assumed to be the maximum (open circles; shaded region is $<0.3$ ). This criterion was used to define the range of valid models in Figure 8a.

poorly constrained values of $c, \varphi_{m}$, and $K_{m}$ in the upper oceanic crust, we did not attempt to estimate $P_{e p}$ but instead we considered its entire range as shown in Figure 8a. While the $P$ wave anisotropy is a function of $\gamma$ at high $P_{e p}$ (where the equant porosity has no effect) and independent of $\gamma$ at low $P_{e p}$ (where fluid flows easily into the surrounding pores), the shear wave anisotropy is independent of $\gamma$ for all $P_{e p}$. The predicted anisotropy at the high and low extremes of $P_{e p}$ is equal to the results for isolated wet and dry cracks, respectively [Hudson, 1981]. The low extreme of $P_{e p}$, 
isolated dry cracks, is also the end-member condition for interconnected aligned cracks that allow completely free fluid flow [Hudson et al., 1996]. This condition may be applicable near the seafloor where the fluids in the aligned cracks and fissures are fully connected to the ocean.

[20] We estimated the range of realistic $P$ wave anisotropy factors in Figure 8a by identifying the conditions for which the $\cos (4 \theta)$ azimuthal velocity variation was low. This constraint was based on the absence of any $\cos (4 \theta)$ variation in the $P$ wave travel times [Barclay et al., 1998]. Figure 8b shows the relative amplitudes of $\cos (2 \theta)$ and $\cos (4 \theta)$ variation predicted by EQUANT ALIGN for the range of models represented in Figure $\overline{8} \mathrm{a}$. In order to account for the possibility that some $\cos (4 \theta)$ signal was present but was mapped into heterogeneity, we assumed that the maximum amplitude of $\cos (4 \theta)$ signal that could be present in our data was 0.3 times the $\cos (2 \theta)$ signal. The ranges of $P_{e p}$ and $\gamma$ that fulfill this condition are identified in Figure 8. The same region in Figure 8a corresponds to a valid range of $P$ wave anisotropy factors of $150-250$, or $1.5-2.5$ times greater than the $S$ wave anisotropy factor. For isolated, fluid-filled cracks, this ratio would be $0-1$ and is consistent with the value of 0.5 quoted by Crampin [1994].

[21] The total delay between two depths $z 1$ and $z 2$ for a vertically traveling shear wave is given by,

$$
\int_{z 1}^{z 2} \frac{\xi(z)}{V_{\max }(z)(1-\xi(z))} d z
$$

where $V_{\max }(z)$ is the fast $S$ wave velocity structure and $\xi(z) *$ 100 is the percent shear wave anisotropy (the anisotropy factor times the percent $P$ wave anisotropy). $V_{\max }(z)$ is used because the first $S$ wave arrival times were used in the inversion for the $S$ wave velocity structure [Barclay et al., 2001]. We calculated the maximum amount of $S$ wave delay that could be attributed to the $P$ wave anisotropy by evaluating equation (2) between depths of $500 \mathrm{~m}$ and $2 \mathrm{~km}$ using the minimum anisotropy factor ratio of 150 . By this approach, we estimate the total shear wave delay due to the observed $P$ wave anisotropy to be $<10 \mathrm{~ms}$. The delay times we measure are 3-15 times greater than this amount, indicating that the $P$ wave anisotropy we detected cannot be a significant source of the observed shear wave anisotropy.

[22] The most likely source for the remaining anisotropy is the shallowmost $500 \mathrm{~m}$ (seismic layer 2A). This inference is consistent with the large variation in split shear wave delays as inferred in the interpretation. For an average delay of $90 \mathrm{~ms}$, the average percent shear wave anisotropy in the shallowmost $500 \mathrm{~m}$ is $18 \%$. For the maximum and minimum delays of 180 and $35 \mathrm{~ms}$, the anisotropy is $30 \%$ and $7 \%$, respectively. The average value is significantly greater than the $10 \%$ limit inferred for competent rock [Crampin, 1994], and is therefore consistent with the extremely low seismic velocities and high porosities of layer $2 \mathrm{~A}$. Because the average wavelength for $S$ waves in layer $2 \mathrm{~A}$ is small $(\sim 120 \mathrm{~m})$, the region sampled by each shear wave arrival is small, and variations in crack density on a similar length scale may give rise to the large variation in delays. A consequence of this interpretation is that seafloor measurements of shear wave delays in the crust are dominated by layer 2A and yield little information on deeper structure. Confirmation of this result requires $P$ wave anisotropy measurements in the shallowmost crust using on-bottom seismic sources.

[23] An alternative explanation is that much of the anisotropy is in the form of isolated, fluid-filled cracks with $\cos (4 \theta)$ azimuthal $P$ wave velocity variation. Small amounts of $\cos (4 \theta)$ variation may be present but not detected by seismic refraction and tomography experiments and because these cracks have a larger effect on the $S$ wave anisotropy than on the $P$ wave anisotropy (Figure 8a), they may indeed be present. The most likely type of isolated, fluid-filled cracks is the EDA microcracks. The presence of EDA microcracks below layer $2 \mathrm{~A}$ does not readily explain the large variation in shear wave delays, however, and it is possible that a combination of microcracks and extensive layer 2A fissuring accounts for most of the shear wave delays.

[24] Seismic anisotropy at this site appears to be pervasive within the uppermost crust and it progressively decreases with depth so that it is either absent or insignificant at depths greater than $3 \mathrm{~km}$. From this we conclude that if the pathways of hydrothermal fluid circulation are influenced by properties that give rise to seismic anisotropy, then any such anisotropic permeabilities are limited to the upper crust. Extensive fissuring in the extrusive layer likely causes strong anisotropic permeability as well as seismic anisotropy. At greater depth, hydrothermal fluids may circulate in aligned pathways that do not give rise to pervasive seismic anisotropy, for example, in fault zones that are widely spaced in comparison with the seismic wavelength.

[25] This study is the first to jointly interpret observations of $P$ and $S$ wave anisotropy at a mid-ocean ridge and to relate them to recent theoretical crack models. An anisotropy study was not the primary aim of the experiment, and better constraints on the range of possible crack distributions may be obtained using near-bottom seismic sources to measure $P$ wave anisotropy in the shallowmost $500 \mathrm{~m}$ beneath the seafloor and a refraction or tomography experiment specifically designed to place better limits on the relative contributions of the $\cos (2 \theta)$ and $\cos (4 \theta)$ components to the azimuthal $P$ wave velocity variation. Improved constraints on the $S$ wave anisotropy would be obtained by a larger number of shear wave splitting observations with earthquake sources that span a range of depths. These conditions may best be met through long-term monitoring of microearthquakes and active source seismic studies at active seafloor hydrothermal systems at slow or intermediate spreading ridges.

\section{Conclusions}

[26] 1. Shear wave splitting, observed at the inner valley floor of the MAR shows a consistent fast polarization directions that is parallel to the trend of the axial valley and split shear wave delays of $35-180 \mathrm{~ms}$.

[27] 2. The anisotropy is consistent with a distribution of vertical cracks that are preferentially aligned perpendicular to the direction of minimum compressive stress. The shear wave anisotropy is also consistent with observations of $P$ wave azimuthal velocity at the same site. 
[28] 3. The anisotropy is likely due to aligned fissures in the uppermost $500 \mathrm{~m}$ and dike-parallel thermal contraction cracks at depths greater than $500 \mathrm{~m}$. EDA cracks may also be present at these depths.

[29] 4. Crack models that explain the observed $P$ wave anisotropy can only explain $5-10 \%$ of the shear wave delays. Shear wave anisotropy in layer $2 \mathrm{~A}$ of $<30 \%$ and/ or an additional distribution of isolated, fluid-filled cracks that was not detected by the $P$ wave study, is required to account for the discrepancy.

[30] Acknowledgments. We thank the Woods Hole Oceanographic Institution OBS group and the captains and crews of the R/V Ewing and the $\mathrm{R} / \mathrm{V}$ Oceanus. The Associate Editor, Martha Savage and an anonymous reviewer provided constructive reviews. This work was supported by the National Science Foundation under grants OCE-9018389 and OCE9530146.

\section{References}

Almendros, J., A. H. Barclay, W. S. D. Wilcock, and G. M. Purdy, Seismic anisotropy of the shallow crust at the Juan de Fuca Ridge, Geophys. Res. Lett., 27, 3109-3112, 2000.

Babuska, V., and M. Cara, Seismic Anisotropy in the Earth, 217 pp., Kluwer Acad., Norwell, Mass., 1991.

Ballard, R. D., and T. H. van Andel, Morphology and tectonics of the inner rift valley at lat. 36N50 on the Mid-Atlantic Ridge, Geol. Soc. Am. Bull., $88,507-530,1977$.

Barclay, A. H., D. R. Toomey, and S. C. Solomon, Seismic structure and crustal magmatism at the Mid-Atlantic Ridge, $35^{\circ} \mathrm{N}, J$. Geophys. Res., 103, 17,827-17,844, 1998 .

Barclay, A. H., D. R. Toomey, and S. C. Solomon, Microearthquake characteristics and crustal $V_{P} / V_{S}$ structure at the Mid-Atlantic Ridge, $35^{\circ}$ N, J. Geophys. Res., 106, 2017-2034, 2001.

Crampin, S., Seismic-wave propagation through a cracked solid: Polarization as a possible dilatency diagnostic, Geophys. J. R. Astron. Soc., 53, 467-496, 1978 .

Crampin, S., Suggestions for a consistent terminology for seismic anisotropy, Geophys. Prospect., 37, 753-770, 1989.

Crampin, S., A review of the effects of crack geometry on wave propagation through aligned cracks, Can. J. Explor. Geophys., 29, 3-17, 1993.

Crampin, S., The fracture criticality of crustal rocks, Geophys. J. Int., 118, 428-438, 1994.

Detrick, R. S., D. R. Toomey, and J. A. Collins, Three-dimensional upper crustal heterogeneity and anisotropy around Hole 504B from seismic tomography, J. Geophys. Res., 103, 30,485-30,504, 1998.

Dunn, R. A., and D. R. Toomey, Crack-induced seismic anisotropy in the oceanic crust across the East Pacific Rise $\left(9^{\circ} 30^{\prime} \mathrm{N}\right)$, Earth Planet. Sci. Lett., 189, 9-17, 2001.

Evans, J. R., G. R. Foulger, B. R. Julian, and A. D. Miller, Crustal shearwave splitting from local earthquakes in the Hengill triple junction, southwest Iceland, Geophys. Res. Lett., 23, 455-458, 1996.

Evans, R., Effects of the free surface on shear wavetrains, Geophys. J. R. Astron. Soc., 76, 165-172, 1984.

Gracia, E., D. Bideau, R. Hekinian, and Y. Lagabrielle, Detailed geological mapping of two contrasting second-order segments of the Mid-Atlantic Ridge between Oceanographer and Hayes fracture zones $\left(33^{\circ} 30^{\prime} \mathrm{N}-\right.$ $\left.35^{\circ} \mathrm{N}\right)$, J. Geophys. Res., 104, 22,903-22,921, 1999.

Hekinian, R., F. Pineau, S. Shilobreeva, D. Bideau, E. Gracia, and M. Javoy, Deep sea explosive activity on the Mid-Atlantic Ridge near $34^{\circ} 50^{\prime} \mathrm{N}$ : Magma composition, vesicularity and volatile content, J. Volcanol. Geotherm. Res., 98, 49-77, 2000.

Hudson, J. A., Wave speeds and attenuation of elastic waves in material containing cracks, Geophys. J. R. Astron. Soc., 64, 133-150, 1981.

Hudson, J. A., E. Liu, and S. Crampin, The mechanical properties of materials with interconnected cracks and pores, Geophys. J. Int., 124, $105-112,1996$.
Hung, S.-H., and D. W. Forsyth, Anisotropy in the oceanic lithosphere from the study of local intraplate earthquakes on the west flank of the southern East Pacific Rise: Shear wave splitting and waveform modeling, J. Geophys. Res., 104, 10,695-10,717, 1999.

Hussenoeder, S. A., Seismic and magnetic constraints on the structure of upper oceanic crust at fast and slow spreading ridges, Ph.D. thesis, Woods Hole Oceanogr. Inst./Mass. Inst. of Technol. Joint Program, Woods Hole, 1998.

Jurkevics, A., Polarization analysis of three-component array data, Bull. Seismol. Soc. Am., 78, 1725-1743, 1988.

Kleinrock, M. C., and S. E. Humphris, Structural asymmetry of the TAG rift valley: Evidence from a near-bottom survey for episodic spreading, Geophys. Res. Lett., 23, 3439-3442, 1996a.

Kleinrock, M. C., and S. E. Humphris, Structural control on sea-floor hydrothermal activity at the TAG active mound, Nature, 382, 149153, 1996b.

Kong, L. S. L., S. C. Solomon, and G. M. Purdy, Microearthquake characteristics of a mid-ocean ridge along-axis high, J. Geophys. Res., 97, 1659-1685, 1992.

Menke, W., B. Brandsdottir, S. Jakobsdottir, and R. Stefansson, Seismic anisotropy in the crust at the Mid-Atlantic plate boundary in south-west Iceland, Geophys. J. Int., 119, 783-790, 1994.

Munson, C. G., C. H. Thurber, Y. Li, and P. G. Okubo, Crustal shear wave anisotropy in southern Hawaii: Spatial and temporal analysis, J. Geophys. Res., 100, 20,367-20,377, 1995.

Nishizawa, O., Seismic velocity anisotropy in a medium containing oriented cracks - transversely isotropic case, J. Phys. Earth., 30, 331 $347,1982$.

Nur, A., and G. Simmons, Stress-induced velocity anisotropy in rock: An experimental study, J. Geophys. Res., 74, 6667-6674, 1969.

Nuttli, O., and J. D. Whitmore, On the determination of the polarization angle of the $S$ wave, Bull. Seismol. Soc. Am., 52, 95-107, 1962.

O'Connell, R. J., and B. Budiansky, Seismic velocities in dry and saturated cracked solids, J. Geophys. Res., 79, 5412-5426, 1974.

Peacock, S., S. Crampin, D. C. Booth, and J. B. Fletcher, Shear wave splitting in the Anza seismic gap, southern California: Temporal variations as possible precursor, J. Geophys. Res., 93, 3339-3356, 1988.

Pointer, T., E. Liu, and J. A. Hudson, Seismic wave propagation in cracked porous material, Geophys. J. Int., 142, 199-231, 2000.

Savage, M. K., X. R. Shih, R. P. Meyer, and R. C. Aster, Shear-wave anisotropy of active tectonic regions via automated $S$-wave polarization analysis, Tectonophysics, 165, 279-292, 1989.

Shearer, P., and J. A. Orcutt, Anisotropy in the oceanic lithosphere-theory and observations from the Ngendei seismic refraction experiment in the south-west Pacific, Geophys. J. R. Astron. Soc., 80, 493-526, 1985.

Sohn, R. A., S. C. Webb, J. A. Hildebrand, and B. D. Cornuelle, Threedimensional tomographic velocity structure of upper crust, CoAxial segment, Juan de Fuca Ridge: Implications for on-axis evolution and hydrothermal circulation, J. Geophys. Res., 102, 17,679-17,695, 1997.

Stephen, R. A., Seismic anisotropy in the upper oceanic crust, J. Geophys. Res., 90, 11,383-11,396, 1985.

Thomsen, L., Elastic anisotropy due to aligned cracks in porous rock, Geophys. Prospect., 43, 805-829, 1995.

van Everdingen, D. A., Fracture characteristics of the Sheeted Dike Complex, Troodos ophiolite, Cyprus: Implications for the permeability of oceanic crust, J. Geophys. Res., 100, 19,957-19,972, 1995.

Wright, D. J., Formation and development of fissures at the East Pacific Rise; Implications for faulting and magmatism at mid-ocean ridges, in Faulting and Magmatism at Mid-Ocean Ridges, Geophys. Monogr. Ser., vol. 106, edited by W. R. Buck et al., pp. 137-151, AGU, Washington, D. C., 1998.

Zhang, Z., and S. Y. Schwartz, Seismic anisotropy in the shallow crust of the Loma Prieta segment of the San Andreas fault system, J. Geophys. Res., 99, 9651-9661, 1994.

A. H. Barclay, School of Oceanography, University of Washington, Box 357940, Seattle, WA 98195, USA. (andrew@ocean.washington.edu)

D. R. Toomey, Department of Geological Sciences, University of Oregon, 1272 Geological Sciences, Eugene, OR 97403-1272, USA. (drt@newberry. uoregon.edu) 